\title{
Comparing And Aligning Outcomes of Two Engineering AND TEChNOLOGY Disciplines IN ONTARIO
}

\author{
David R. Waller ${ }^{\text {, Sima Zakani }}{ }^{2}$, Nerissa Mulligan ${ }^{1}$, Brian Frank ${ }^{l}$, \\ Jake Kaupp ${ }^{1}$, Roderick Turner ${ }^{3}$, and Richard Hornsey ${ }^{4}$ \\ ${ }^{1}$ Queen's University, ${ }^{2}$ University of British Columbia, \\ ${ }^{3}$ Seneca College of Applied Arts and Technology, ${ }^{4}$ York University \\ *nerissa.mulligan@queensu.ca,
}

\begin{abstract}
The separate development of engineering and technology programs in Ontario has made transfer between these program types a complicated process. The process often requires assessment on a case-by-case basis and considers different aspects of knowledge, skills, and performance. This study was conducted to determine the level equivalency between two engineering and technology disciplines with the purpose of informing the development of transfer policy and comprehensive bridging programs in the province.

Outcomes, content, and function of engineering and technology programs in Ontario were analyzed using a common framework in two disciplines: mechanical and electrical. Material from 7 engineering and 10 technology programs, including syllabi, learning outcomes, and reports was collected and analyzed, along with publically available information about programs. Slightly less than $40 \%$ of the courses in representative first year Mechanical and first and second year Electrical/Electronics Technology programs had equivalency to courses in engineering degree programs. The level of cognitive process expected for problemsolving outcomes is higher in the engineering programs than technology programs, and vice versa for outcomes related to hands-on skills.

Overall, the analysis indicated sufficient alignment between engineering and technology programs to suggest transfer students may have acquired the necessary skills and knowledge of introductory level courses that are similar in content. Through hybrid bridging subjects and tests on prior knowledge, engineering programs can ensure incoming transfer students meet all CEAB accreditation criteria.
\end{abstract}

Keywords: transfer, program learning outcomes, curriculum mapping, mechanical engineering, mechanical technology, electrical engineering, electrical technology, electronics technology

\section{INTRODUCTION}

Establishing points of equivalency for transfer between engineering and technology programs in Ontario has been a complex process that often requires the assessment of a student's knowledge profile and skills on an individual basis. This problem exists because these programs were initially developed to serve separate needs in the engineering and technology industry without the intention of supporting efficient student transfer between the two [8]. The difference in both outcomes and curricula, despite some similar content, makes it difficult to provide credit for prior learning when transferring. Additionally, stringent engineering accreditation qualifications set out by the Canadian Engineering Accreditation Board (CEAB) make it challenging to accept credit from technology programs into accredited engineering programs [3]. CEAB defines the content that must be covered for accredited engineering programs. Institutions are responsible for ensuring other accreditation criteria is met including elements of the program environment pertaining to the quality of the educational experience, faculty (expertise, competency, professional status), and contact hours.

This situation provides an opportunity to rethink how credit transfer is done in Ontario, perhaps differently from provinces like British Columbia and Alberta that have a stronger link between technology and engineering programs.

The specific goal for this study was to identify the level of similarity of the learning outcomes and course content between engineering and technology programs in the disciplines of electrical engineering/technology and mechanical engineering/technology. Nicole Fallon explains that transfer between engineering and engineering programs is a multifaceted problem that requires investigation of both the content and context of learning [4]. It is important that both the topics covered (content) and the expectations for depth of understanding (context) are aligned to grant transfer credits. For this reason, a framework developed by Zakani et al. (2016) 
was used to capture both elements [9]. This framework considers the equivalency of content, context of learning, and function of courses in the overall curriculum, and was designed to be applicable for transfer into engineering or technology.

\section{METHODS}

Engineering and technology programs were compared using an approach developed by Moskowitz and Stephens for assessing the equivalency of programs [6]. They explain that a comprehensive evaluation can be made on program equivalency through the systematic analysis of four elements:

(i) Content - The fundamental concepts and content that are covered by a program. This addresses the area of concern for content disparity between program types.

(ii) Context - The depth and complexity of tasks and learning outcomes. This addresses what cognitive processes are the focus for development.

(iii) Function - The relationship between courses in the curriculum. This addresses whether programs offer standalone courses or courses that build off ef each other for deeper understanding.

(iv) Structure - The order and sequence of content delivery. This addresses surface level similarities and differences between these program types and order/timing of course delivery.

This method was shown to be effective at capturing differences between fundamental engineering science courses and engineering design courses [8]. To capture all the elements for the systematic analysis of equivalency for Mechanical and Electrical/Electronics at a larger program level, several sources and artifacts were needed. For this analysis specifically, program learning outcomes, and syllabi or course descriptions from each program's curriculum were used as data sources.

\subsection{Program Learning Outcomes}

Program learning outcomes provided insight into the content and context of each program. Specifically, the Ontario Qualification Framework was used to determine the learning outcomes for technology programs. For engineering programs, learning outcomes were obtained by contacting engineering programs and asking permission to share their CEAB program level indicators. Program learning outcomes were also obtained from publicly available resources.

Bloom's Taxonomy of Educational Objectives was used to measure the complexity of learning that students are expected to demonstrate in a given program or course, as defined by the program learning outcomes [2].
Bloom's was adopted because it is a common framework used to assess learning outcomes. It can be applied when information on other courses or activities in a program is limited, and the language has a high degree of overlap with typical language used to describe explicit learning outcomes. Using Anderson and Krathwohl's revision of Bloom's Taxonomy, this study focused on the cognitive process aspect of the cognitive domain [1]. The following levels describe the different cognitive processes in the framework:

(i) Remember - Retrieve and recall knowledge from long term memory.

(ii) Understand - Construct meaning from instructions and demonstrate acumen.

(iii) Apply - Use appropriate procedures or processes in a given situation.

(iv) Analyze - Break down problems into parts to determine how they relate to one another, and use the parts to construct an overall solution.

(v) Evaluate - Make decisions based on standards or criteria.

(vi) Create - Put elements together to form a coherent whole.

To distinguish between the cognitive process expectations levels of learning, learning outcomes were categorized by five curriculum components known as the CEAB Accreditation Units (AU). The components and examples of topics include [3]:

(i) Mathematics - calculus, linear algebra, numerical analysis, probability

(ii) Natural Science - physics, chemistry, earth science, life science

(iii) Engineering Science - modelling, simulation, thermodynamics, fluid mechanics, electric/electronics circuits, engineering drawing

(iv) Engineering Design - creative/iterative design, open-ended problem solving, handson skills, critical thinking, project management

(v) Complementary - engineering economics, technical communications, law and ethics, health and safety

Though technology programs are not required to cover all five components, they provided a point of comparison for program structure. Based on these frameworks, the learning outcomes were analyzed in four steps:

1) Assign a CEAB $\mathrm{AU}$ content area to the learning outcome

2) Identify the nomenclature that describes the topics covered in the AU 
3) Isolate the action verb of the learning outcome

4) Associate a cognitive process to the action verb

\subsection{Course Descriptions}

Course descriptions were used to analyze the content and structure of programs. For transfer between engineering technology and engineering programs, or vice versa, one of the first steps is to identify the similarities and differences in content delivery. Kopera-Frye et al. assert that curriculum mapping is a versatile tool for comparing the quality of teaching and learning in higher education [5]. Plaza et al. also highlight that curriculum mapping can provide a visualizing of the courses, how they are related, and the timeline of a program [7]. For both analyses, $70 \%$ content similarity was used to determine equivalency between courses.

Queen's University and Seneca College curriculums were used as benchmarks for comparison with the other programs included in the analyses. The courses from ten institutions offering both Mechanical Technology and Electrical/Electronics Technology programs were mapped to courses in the Queen's Mechanical and Electrical Engineering curriculums. Additionally, the courses in the Mechanical and Electrical/Electronics Engineering curriculums of seven=institutions were mapped to technology program courses at Seneca College. Data was obtained by contacting engineering and technology programs and instructors and asking permission to share their course descriptions and syllabi. Online course descriptions were used when syllabi were not available.

\section{RESULTS}

\subsection{Comparisons of Program Learning Outcomes}

Analysis of program learning outcomes from engineering and technology programs indicated differences between task expectations (context) and skill emphasis (content). Results were visualized using treemaps which display the relationships between common structures as sets of hierarchical (Macro-MesoMicro) nested rectangles. In this analysis, the first three steps corresponded to the levels of hierarchy, where learning outcomes were first grouped according to their AU category. Within their AU category groupings, learning outcomes were separated by AU topic followed by the learning outcome action verb. Sample treemaps for Mechanical Engineering and Mechanical Technology program learning outcomes can be seen in Figure 1 and Figure 2, respectively.

For Mechanical Engineering programs, results show that institutions tend to emphasize Engineering Science,
Engineering Design, and Complementary content areas in the first two years of study. These programs focus on topics such as physics, modelling, materials science, statics and solid mechanics, thermodynamics, and fluid mechanics. Conversely, learning outcomes for the first two years of Mechanical Technology programs concentrate on Complementary studies, followed by Engineering Design and Engineering Science. For Mechanical Technology curriculums, Complementary studies involve understanding and following predefined codes and safety practices, and topics in Engineering Design and Engineering Science emphasize engineering drawing, materials sciences, and basic sciences.

From the analysis of Electrical Engineering program learning outcomes, results indicate that institutions tend to offer curriculums focusing on Engineering Science, Complementary, and Engineering Design. Electrical/Electronics Technology programs place a higher priority on Engineering Design, Complementary studies, and Engineering Science content areas. Furthermore, differences between engineering and technology program learning outcomes within the same $\mathrm{AU}$ content category were present. For example, it is important to note that Engineering Design topics in representative engineering program learning outcomes emphasize development of problem solving skills while technology programs focus on hands-on skills.

A general verb comparison between engineering and technology programs for all action verbs in learning outcomes of both disciplines was completed and is displayed in Figure 3. From the figure, it can be seen that both engineering and technology program learning outcomes are associated with a wide variety of cognitive processes. However, it appears that engineering programs have a larger proportion of higher cognitive processes when compared with technology programs, particularly in the category of engineering design and engineering science. It is also evident that technology programs use a wide range of verbs to describe their learning outcomes.

\subsection{Curriculum Mapping}

Before comparing courses in engineering and technology programs, curriculum mapping was performed between Queen's University Mechanical Engineering and Electrical Engineering, and other Mechanical Engineering and Electrical Engineering programs at the representative Ontario institutions. This analysis indicated $78 \%$ and $86 \%$ of courses in first and second year engineering at Queen's University are covered by courses in other Mechanical Engineering programs. For Electrical Engineering, 73\% and $60 \%$ of courses in the first and second year of the Queen's University curriculum are covered by 


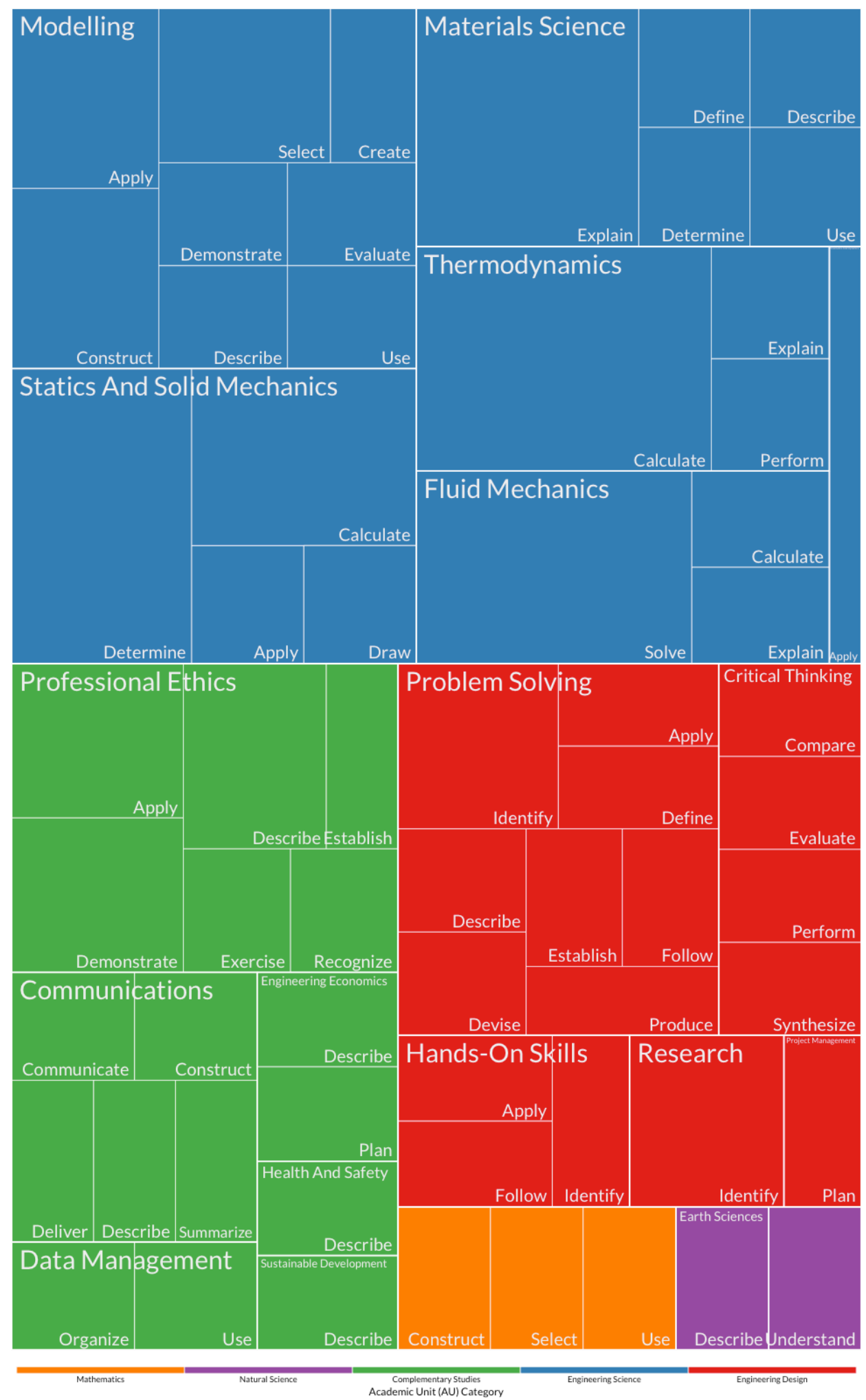

Fig. 1. Treemap of program learning outcomes for a representative Mechanical Engineering program. CEAB AU categories are: Mathematics (orange), Natural Sciences (purple), Complementary Studies (green), Engineering Science (Blue), and Engineering Design (Red). 


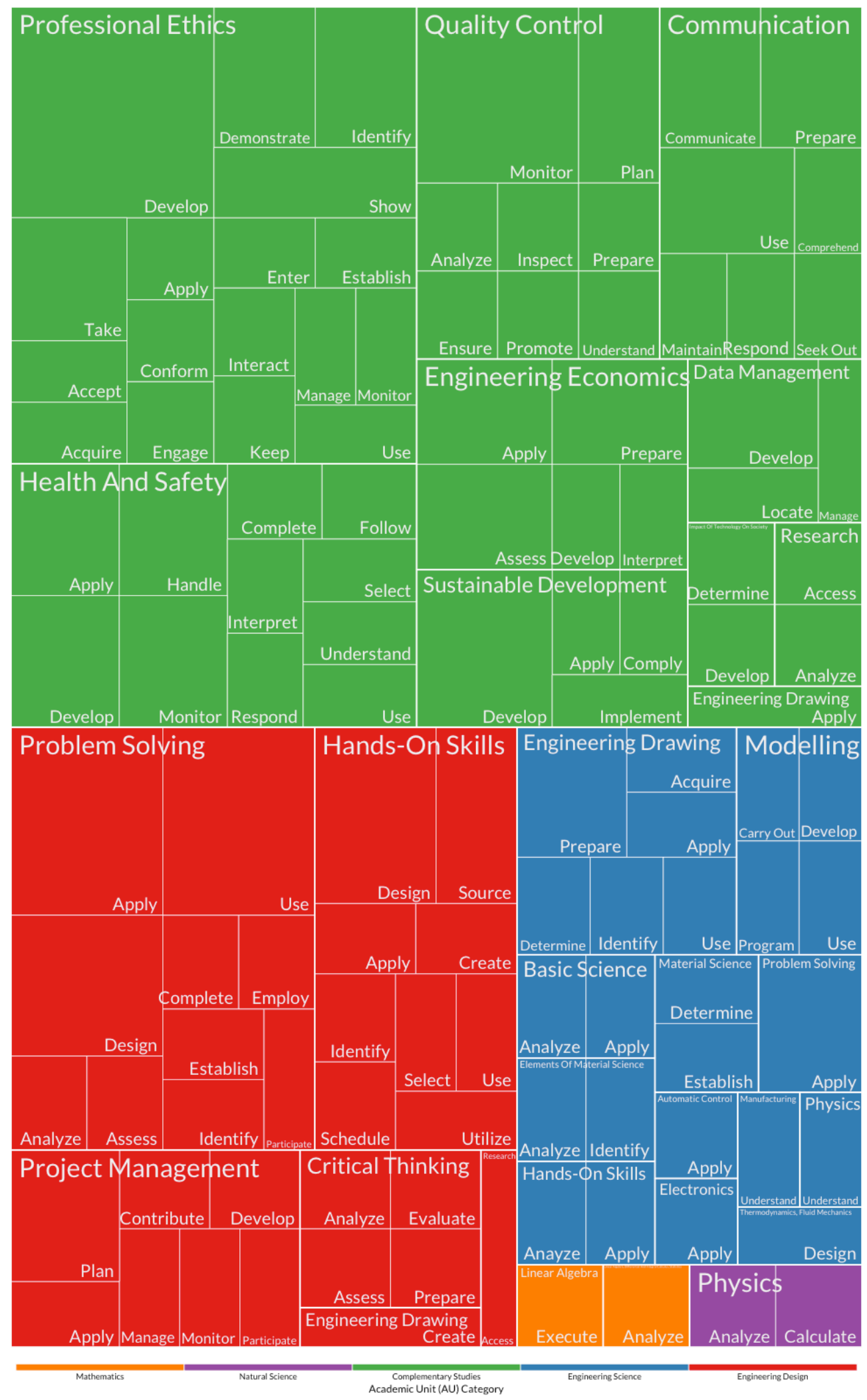

Fig. 2. Treemap of program learning outcomes for a representative Mechanical Technology program. CEAB AU categories are: Mathematics (orange), Natural Sciences (purple), Complementary Studies (green), Engineering Science (Blue), and Engineering Design (Red). 
Proc. 2017 Canadian Engineering Education Association (CEEA17) Conf.

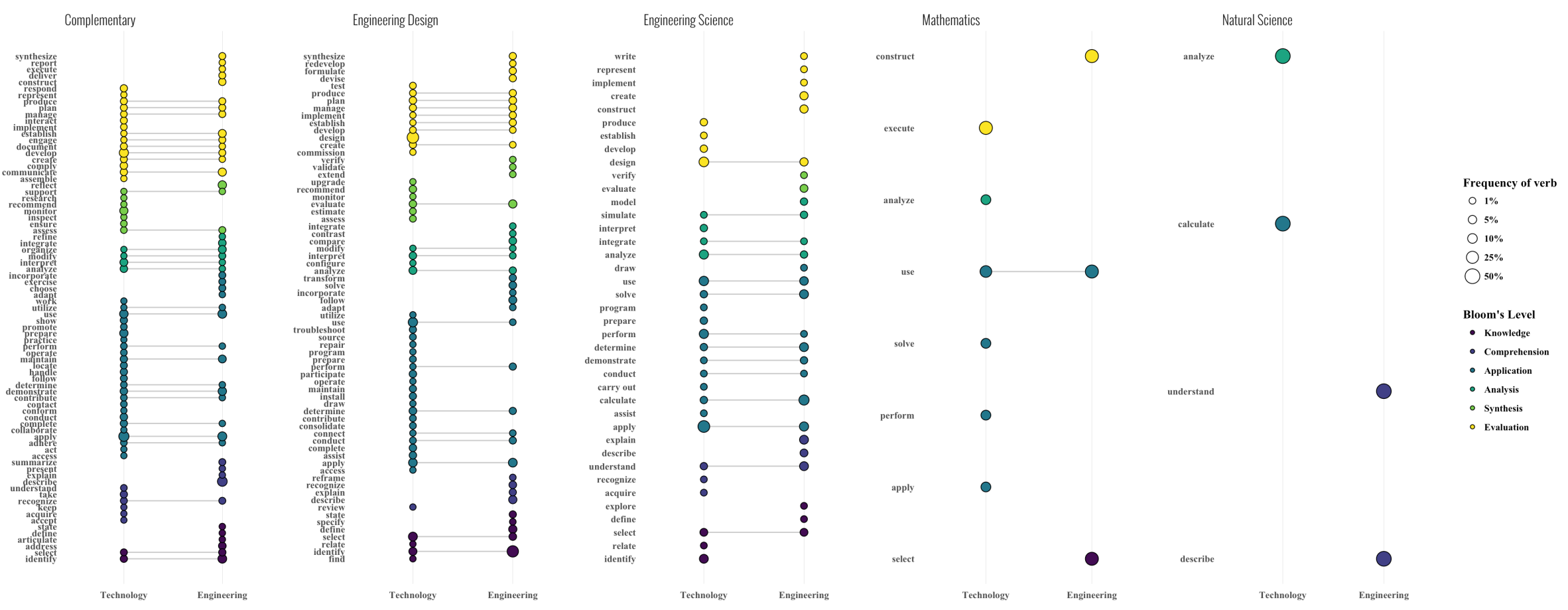

Fig. 3. Comparison of action verbs in program learning outcomes for engineering and technology programs. 
curriculums of other Electrical Engineering programs. This high level of mapping confirmed the equivalency of engineering programs across various institutions in Ontario.

Curriculum mapping for Queen's University Mechanical Engineering to other Mechanical Technology programs show that only $30 \%$ and $55 \%$ of courses in the first and second year of Queen's are covered in the technology programs. Results from the curriculum mapping of Queen's University Electrical Engineering to other Electrical/Electronics Engineering Technology programs reveal only $33 \%$ and $38 \%$ of courses in the first and second year of Queen's University's curriculum are covered by courses in the engineering technology programs.

For consideration of transfer from engineering to technology programs, curriculum mapping was performed using Seneca College as a benchmark for technology programs. Interestingly, for Seneca's Mechanical Technology program, only $37 \%$ and $19 \%$ of courses in the first and second year were covered by Mechanical Engineering programs. Electrical Engineering programs have a much higher level of mapping with coverage of $40 \%$ and $63 \%$ of courses in the first and second year of Seneca's Electronics Technology program.

\section{DISCUSSION AND CONCLUSIONS}

The analysis of program learning outcomes indicates that engineering programs have more emphasis on skills related to design including problem solving, developing models, and using models. This indicates a demand for higher cognitive levels associated with design activities. Technology programs have a heavier emphasis on developing hands-on skills not common in engineering programs such as troubleshooting, testing, installing, and maintaining. These skills are associated with mid-level cognitive processes. This distinction is clearly illustrated in the use of modelling in the study of engineering and technology. Engineering programs teach their students to develop and validate models, while technology programs focus on developing skills that relate to selecting and applying appropriate models. The selection and application of models and procedures results in a higher focus on topics of quality control, codes and standards, and health and safety which is evident in technology program learning outcomes. This finding may be a result of preparing students for different work environments.

For curriculum mapping, the use of Queen's University as a benchmark was validated by the high level of mapping between Queen's and the other engineering programs at the representative institutions for both disciplines. From the analysis, less than $40 \%$ of the courses in representative first year Mechanical and first and second year Electrical/Electronics Technology programs had equivalency to courses in engineering degree programs. Though some specific technology programs in both disciplines exhibited more than $50 \%$ coverage in the first two years, the majority of technology programs had lower levels of mapping. This suggests that in terms of content, only certain introductory level courses will have equivalency (typically courses in calculus, discipline specific engineering science, and complementary), and consideration of the specific institution when a student is transferring is warranted. Furthermore, the differences between Mechanical and Electrical/Electronics Technology programs highlights the need for discipline specific design transfer strategies.

It was interesting to note the higher level of mapping for second year Mechanical Technology. This may be a result of engineering programs covering a wide range of engineering science topics in first year while discipline specific courses begin in second year. Technology programs tend to have a discipline specific focus throughout the entire curriculum, hence more coverage of a second year engineering degree. Additionally, institutions should be aware that coverage of first and second year courses in engineering curriculums may have a contribution from courses in third year technology curriculums.

Limitations of the study include the number of institutions involved, access to clear and complete course descriptions and syllabi, and the language used to describe program learning outcomes. In particular, it was clear from the analysis that learning outcomes from technology programs are often meticulously written, while engineering programs tend to be more generic. However, engineering programs have only recently started using learning outcomes on a wide scale, and it is expected that learning outcomes will improve quickly to clearly and accurately reflect course learning.

Overall, the combined analysis of program learning outcomes and curriculum mapping demonstrates some level of alignment between engineering and technology programs in the first two years of study. Though the emphasis of cognitive skills may differ, for course content that matches, there are enough similarities to suggest students may have acquired the necessary understanding and skills expected in introductory level courses. Therefore, for students transferring within their respective discipline, a Prior Learning Assessment and Recognition (PLAR) can be administered where students are given tasks to evaluate missing skills, similar to the process currently used in British Columbia. With this approach, programs can develop appropriate paths of study for incoming students, and those who have demonstrated proficiency in the PLAR can then focus on the courses that were not covered in their previous program.

Furthermore, the conclusions from this study inform the development of bridging programs which can focus on addressing the gaps in skills and knowledge of incoming transfer students. By ensuring that hybrid bridge subjects 
cover all necessary content and program environment requirements, students will be able to complete the remainder of their degrees while still meeting $\mathrm{CEAB}$ accreditation criteria.

\section{Acknowledgements}

The authors would like to acknowledge ONCAT for funding this research. As well, the authors would like to thank the institutions involved in the study and all the programs and individuals who provided data.

\section{References}

[1] Lorin W. Anderson, and David R. Krathwohl, A taxonomy for learning, teaching, and assessing: A revision of Bloom's taxonomy of educational objectives. New York, NY: Pearson, 2000 \{ISBN: 978-0321084057\}

[2] Benjamin S. Bloom, Max D. Englehart, Edward J. Furst, Walter H. Hill, and David R. Krathwohl, Taxonomy of educational goals. Handbook I: Cognitive Domain. New York, NY: McKay, 1956 \{ISBN: 978-0679302094\}

[3] Engineers Canada. Canadian engineering accreditation board (CEAB): 2016 Accreditation criteria and procedures. 2017 https://engineerscanada.ca/sites/default/files/accreditationcriteria-procedures-2016-final.pdf
[4] Nicole Fallon, Leaning outcomes in credit transfer: A key tool for innovation in student mobility. Toronto, ON: Ontario Council on Articulation and Transfer (ONCAT), 2015.

[5] Karen Kopera-Frye, John Mahaffy, Gloria Messick Svare, "The map to curriculum alignment and improvement". Collected Essays on Learning and Teaching, vol. 1 pp. 8-14, 2008.

[6] Jay Moskowitz, and Maria Stephens, Comparing Learning Outcomes: International Assessment and Education Policy. New York, NY: Routledge-Falmer, 2004 \{ISBN: 0415304199\}

[7] Cecilia M. Plaza, JoLaine Reierson Draugalis, Marion K. Slack, Grant H. Skrepnek, Karen Ann Sauer, "Curriculum mapping in program assessment and evaluation," American Journal of Pharmaceutical Education, vol. 71 no. 2, article 20, 2007.

[8] David R. Waller, Sima Zakani, Nerissa Mulligan, Brian Frank, Jake Kaupp, Roderick Turner, and Richard Hornsey, Comparing and Aligning Outcomes of Two Engineering and Technology Disciplines in Ontario. Toronto, ON: Ontario Council on Articulation and Transfer (ONCAT), March 2017.

[9] Sima Zakani, Brian Frank, Roderick Turner, and Jake Kaupp, Framework for transferability between engineering and technology programs. Toronto, ON: Ontario Council on Articulation and Transfer (ONCAT), March 2016. 